\title{
SURFACE EFFECTS IN Fe-BASED NANOCRYSTALLINE ALLOYS
}

A. ŚlawSKa-WANIEWSKa

Institute of Physics, Polish Academy of Sciences

Al. Lotników 32/46, 02-668 Warszawa, Poland

K. BRzózKa

Department of Physics, Technical University, Malczewskiego 29, 26-600 Radom, Poland

AND J.M. GRENEChe

Equipe de Physique de l'Etat Condensé, URA CNRS 807, Université du Maine 72017 Le Mans, France

The microstructural and Mössbauer investigations of $\mathrm{FeZrBCu}$ nanocrystalline alloy are presented. The results obtained indicate that fine bcc-Fe grains do possess identifiable surface properties which arise from the symmetry restriction at grain boundary.

PACS numbers: 75.50.Kj, 76.80.+y, 61.16.Bg

Nanocrystalline $\mathrm{Fe}-\mathrm{Zr}-\mathrm{B}$ alloys produced by controlled crystallization of the amorphous ribbons show simultaneously good soft magnetic properties and large saturation magnetization [1-3]. The intergranular phase, formed by the amorphous material deficient in iron (due to precipitation of bcc-Fe crystalline phase) affects the exchange coupling between the grains and, consequently, the behavior of such nanocomposite material. It has recently been shown that the amorphous $\mathrm{FeZrB}(\mathrm{Cu})$ alloys exhibit the Invar type compositional characteristics [4] (e.g. the decrease in the Curie temperature and the room temperature saturation magnetization with increasing $\mathrm{Fe}$ concentration) and thus an enhancement of the exchange interactions in the system with evolution of the precipitation process can be achieved.

It is known that magnetic properties depend on the microstructure of such nanocomposite material and are particularly sensitive to the grain size and the grain boundary structure [6]. Nanocrystals have typically the size 10-15 $\mathrm{nm}$ and hence a large fraction of their atoms are the surface atoms. It may thus be expected that the grains have surface properties which differ from those of the interior because of the symmetry restriction at grain boundary which leads to a concept of 
"spin-glass" type disorder of the surface spins [5], surface anisotropy [6] and magnetostriction [6]. The important role of the surface magnetostriction has recently been confirmed experimentally in $\mathrm{Fe}-\mathrm{Zr}-\mathrm{B}$ nanocrystalline materials [6]. The aim of this paper is to study the surface effects and the properties of the intergranular amorphous phase in the nanocrystalline system on a basis of complementary microstructural and Mössbauer investigations.

$\mathrm{Fe}_{85} \mathrm{Zr}_{7} \mathrm{~B}_{6} \mathrm{Cu}_{2}$ amorphous ribbon was produced by the melt-spinning method in an argon atmosphere. Annealing of the material at $540^{\circ} \mathrm{C}$ for 1 hour results in creation of the bcc-Fe phase, as shown by X-ray diffraction studies [3]. The microstructure of the annealed material was studied by means of the transmission electron microscopy (TEM) and the bright-field micrograph as well as the corresponding electron diffraction pattern is presented in Fig. 1. It is seen that the

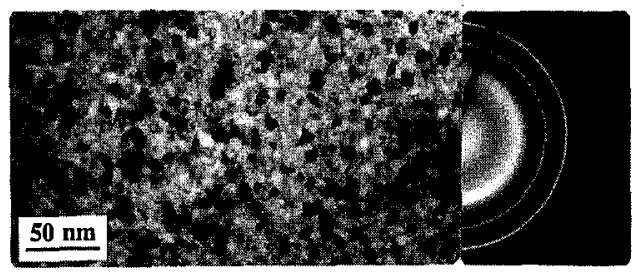

Fig. 1. TEM micrograph and diffraction pattern of $\mathrm{FeZrCuB}$ nanocrystalline alloy.

sample shows an ultrafine structure of crystalline grains (with the average grain diameter of about $10 \mathrm{~nm}$ ) embedded in the residual amorphous matrix. The electron diffraction pattern consists of a mixture of sharp ring patterns diffracted by randomly distributed bcc-Fe crystals and a diffuse ring characteristic of the amorphous phase. The volumetric fraction of the crystalline phase has been estimated by the magnetic methods from the temperature dependence of saturation magnetization [3]. Since the Curie temperature of the amorphous matrix is much lower than that of the crystalline $\alpha$-Fe phase, thus, at elevated temperatures only ferromagnetic grains contribute to the effective saturation magnetization of the sample. Assuming that the magnetization of fine particles is the same as that of bulk iron, the crystalline fraction in the sample studied was estimated to be $67 \%$.

The Mössbauer spectroscopy, due to its local probe, is one of the most suitable experimental technique which may be applied to identify different magnetic phases in multiphase granular materials [6], among others in Fe-based nanocrystalline alloys of the type considered [7]. The Mössbauer measurements were performed at room temperature using a conventional constant acceleration spectrometer with ${ }^{57} \mathrm{Co} / \mathrm{Rh}$ source. The spectrum obtained in the as-quenched $\mathrm{FeZrBCu}$ alloy, showing a broad sextet characteristic of topologically disordered material, is presented in Fig. 2a and the corresponding distribution of the hyperfine field $P(H)$ in Fig. 2b. The obtained $P(H)$ curve is very broad and can be separated into two Gaussian components corresponding to low-field and high-field contributions. Such a bimodal structure can be attributed to local magnetic environments of: (i) Fe-rich region (low-field part) in which a certain distribution of $\mathrm{Fe}-\mathrm{Fe}$ near 


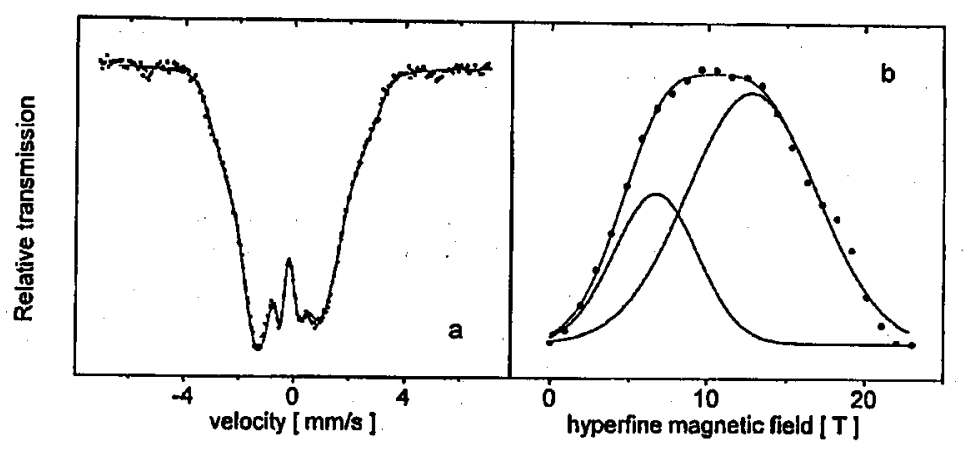

Fig. 2. Mössbauer spectrum (a) and related $P(H)$ curve (b) in amorphous sample.

neighbors distances leads to competing ferro- and antiferromagnetic interactions and (ii) Fe-poor region (high-field part) in which $\mathrm{Fe}$ atoms are partially coordinated with $\mathrm{Zr}, \mathrm{B}$ and $\mathrm{Cu}$.

Annealing of the amorphous materials at $540^{\circ} \mathrm{C}$ for 1 hour leads to a drastic change of the Mössbauer spectra and to the appearance of a hyperfine sextet with very sharp lines; the spectrum, in a form of experimental points, is shown in Fig. 3. In the first approach this spectrum has been fitted assuming the superimposition

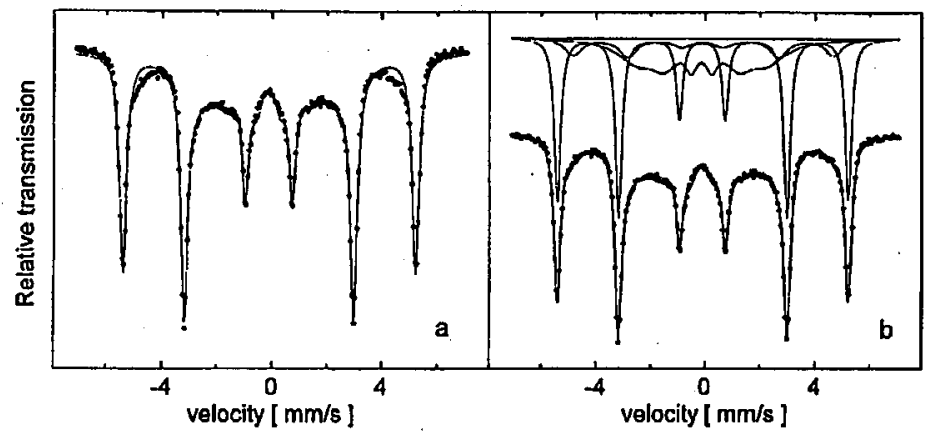

Fig. 3. Mössbauer spectrum (points) of nanocrystalline FeZrBCu alloy; the solid lines represent the fit of two contributions (a) and three contributions (b).

of the broad component attributed to residual amorphous matrix and the well defined sextet of the crystalline phase. The fitted curve is shown as a solid line in Fig. 3a. Although the hyperfine parameters obtained for the crystalline phase were characteristic of bcc Fe (as it was expected from X-ray diffraction study), nevertheless the deviation of the fitted curve from the experimental one indicates that another contribution, which appears as a shoulder of the outermost lines attributed to crystalline phase at lower fields, has to be taken into account. The Mössbauer spectrum of the nanocrystalline sample can therefore be decomposed into three subspectra (shown in Fig. 3b): (1) inner sextet of broad and overlapping lines with the average value of the hyperfine field $\left\langle\mathrm{H}_{\mathrm{hf}}(\mathrm{am})\right\rangle=14.7 \mathrm{~T}$ and the isomer shift 
$\sigma(\mathrm{am})=-0.14 \mathrm{~mm} / \mathrm{s}$ originating from the residual amorphous matrix deficient in iron, (2) well resolved sextet of the crystalline phase with sharp lines and hyperfine parameters $H_{\mathrm{hr}}(\mathrm{cr})=33.08 \mathrm{~T}$ and $\sigma(\mathrm{cr})=-0.107 \mathrm{~mm} / \mathrm{s}$, i.e. the values typical of bcc Fe, and (3) a sextet exhibiting broad lines with $\left\langle H_{\mathrm{hf}}(\mathrm{s})\right\rangle=29.6 \mathrm{~T}$ and $\sigma(\mathrm{s})=-0.09 \mathrm{~mm} / \mathrm{s}$ that is with the average value of the hyperfine field lower than that for the bcc Fe but significantly higher than that in the amorphous matrix. It is worth noting that the hyperfine parameters obtained for the third component are neither typical of $\mathrm{Fe}-\mathrm{Zr}$ nor $\mathrm{Fe}-\mathrm{B}$ crystals which can be considered as additional crystallization products which may eventually appear during annealing. Since it has already been shown that in $\mathrm{FeZrBCu}$ nanocrystalline systems a large surface-to-volume ratio affects significantly such magnetic properties as the effective magnetostriction [4], it may be expected that the third component originates from the atoms placed at the grain surfaces and/or crystal-amorphous interfaces. This conclusion can additionally be supported by the consideration of the relative contribution of different components to the Mössbauer spectrum. It has been found that the volumetric fraction of the amorphous (1), crystalline (2) and surface (3) phases are $33 \%, 52 \%$ and $15 \%$, respectively. Considering that crystalline fraction estimated from the bulk magnetization measurements, where the grains together with their surfaces contribute to the saturation magnetization, is about $67 \%$, a nice agreement with the Mössbauer data is obtained when the crystalline (2) and surface (3) contributions are taken into account. The special properties of the grain surfaces, different from the bulk one, arise from the broken symmetry at grain boundary, lower coordination of the Fe surface atoms than that inside the grain and a possible bonding of these atoms to the matrix.

\section{References}

[1] K. Suzuki, A. Makino, N. Kataoka, A. Inoue, Mater. Trans. JIM 32, 93 (1991).

[2] K. Suzuki, A. Makino, A. Inoue, T. Masumoto, J. Appl. Phys. 74, 3316 (1993).

[3] A. Ślawska-Waniewska, P. Nowicki, Mater. Sci. Forum 179-181, 563 (1995).

[4] A. Ślawska-Waniewska, R. Żuberek, J. Magn. Magn. Mater., in press.

[5] R. Kodama, C. Seaman, A. Berkowitz, M. Maple, J. Appl. Phys. 75, 5639 (1994).

[6] A.H. Morrish, in: Studies of Magnetic Properties of Fine Particles, Ed. J.L. Dormann, Elsevier, 1992, p. 181.

[7] I. Navarro, A. Hernando, M. Vazquez, Seon-Cuo Yu, J. Magn. Magn. Mater. 145, 313 (1995). 